\title{
Non-axisymmetric SOL-transport study for tokamaks and stellarators
}

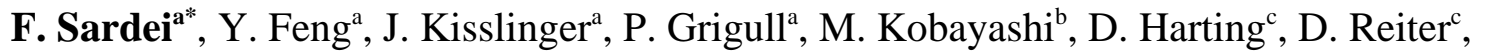 \\ G. Federici ${ }^{\mathrm{d}}$ A. Loarte $^{\mathrm{e}}$ \\ ${ }^{a}$ Max-Planck-Institut für Plasmaphysik, Teilinstitut Greifswald, Euratom Association, 17491 \\ Greifswald, Germany \\ ${ }^{b}$ National Institute for Fusion Science,322-6 Oroshi, Toki, 509-5292, Japan \\ 'Institut für Plasmaphysik, Forschungszentrum Jülich Gmbh, Euratom Association, Trilateral \\ Euregio Cluster, 52425 Jülich, Germany \\ ${ }^{d}$ ITER Garching Joint Work Site, Boltzmannstrasse 2, 85748 Garching, Germany \\ ${ }^{e}$ EFDA Close Support Unit, Boltzmannstrasse 2, 85748 Garching, Germany
}

\begin{abstract}
The paper addresses basic features of non-axisymmetric edge transport induced in tokamaks by local limiters or external magnetic perturbations and in low-shear stellarators by the presence of edge magnetic islands. 3D simulations and, if available for comparison, experimental results are presented and discussed for three devices, ITER during start-up operation, TEXTOR-DED and W7-AS, having edge topologies totally different from each other. The modeling is performed with the EMC3/EIRENE code, which treats self-consistently plasma, neutral and impurity transport in a general 3D Scrape-Off Layer (SOL) with arbitrarily complex geometry of magnetic configuration and plasma-facing components. Shown are code predictions of the power load on the ITER start-up limiters as well as modeling results on the transport in the TEXTOR-DED stochastic edge and on the physics of stable detachment in W7-AS. Experimental observations confirming the code simulations are referenced for both TEXTOR-DED and W7-AS, a direct comparison between modeling and experimental results is shown for W7-AS.
\end{abstract}

PACS: 52.40.Hf, 52.55.Rk, 52.65.-y, 52.65.Pp

JNM keywords: Theory and Modeling

PSI-17 keywords: Edge modeling, Island divertor, ITER, TEXTOR-DED, W7-AS

* Corresponding author address: Boltzmannstr. 2, 85748 Garching, Germany

* Corresponding author e-mail: francesco.sardei@ipp.mpg.de 


\section{Introduction}

In helical devices, SOLs are intrinsically three-dimensional (3D) because of their nonaxisymmetric magnetic field structure. Additionally for island divertors, toroidally continuous recycling is impeded by technically unavoidable discretization of divertor plates [1]. In standard tokamaks, a 3D SOL can be induced by local sources like gasinjection or by non- axisymmetric installations like discrete limiters, as proposed for ITER during the plasma start-up phase [2]. Axisymmetry is also violated by applying external perturbation fields to tokamaks in order to provide an active control of the plasma edge transport by magnetic ergodization, like in TEXTOR-DED [3,4] and TORE SUPRA [5,6], or to mitigate the impact of large ELMs on the power exhaust like in DIII$\mathrm{D}[7,8]$. In all these cases, the edge transport becomes 3D as well and SOL transport models have to be upgraded to meet the increased dimensionality. Such 3D models are needed for understanding and predicting the edge physics of plasma, neutrals and impurities and for optimizing the plasma-facing components. For operating machines, realistic 3D transport models can be used as complementary "3D numerical-diagnostic tools" for the experiment, in view of the technical difficulty to provide sufficient toroidally-resolved diagnostic data. ITER, TEXTOR-DED and W7-AS have been chosen as examples because of their completely different topologies.

\section{ITER}

In ITER, axisymmetry is violated by discrete start-up limiters needed for protecting the wall during the current ramp-up and ramp-down phases of a discharge. The size of the limiters is bounded by port-accessibility and remote-handling constraints, which reduce the total affordable limiter surface to a few $\%$ of the total plasma surface at the edge, leading to high power loads on the limiters. These power loads have to be kept below the technical limits for the envisaged plasma-facing materials, which range between 5 and 8 $\mathrm{MW} / \mathrm{m}^{2}$.

The current design consists of two limiter modules located at opposite toroidal positions. Their small size in the presence of a significant magnetic shear introduces a complex pattern of open field lines having completely different connection lengths $\left(L_{\mathrm{c}}\right)$ ranging from $250 \mathrm{~m}$ (several toroidal turns) to infinity [9]. This topology makes the limiter SOL non-transparent to the adoption of simple transport models, which cannot replace a selfconsistent 3D numerical treatment in the assessment of non-axisymmetric effects. Such a 3D study [9] has been performed with the EMC3-EIRENE code [10,11] for three current ramp-up plasma-equilibrium configurations, 2.5, 4.5 and 6.5 MA, selected from the socalled ITER start-up scenario 2 [12]. The upstream parameters $P_{\mathrm{SOL}}$ and $n_{\text {up }}$ are taken from a core-transport analysis based on the ASTRA code [13]. The geometry of the radial-poloidal domain of the limiter-SOL used in the simulations of the 6.5 MA case is shown in Figs. 1 and the $L_{\mathrm{c}}$ distribution over this domain is plotted in Fig. 2. The $L_{\mathrm{c}}$ picture exhibits a typical sequence of vertical stripes with small connection lengths representing the flux tubes of the limiter shadow, where particles and power are lost to the limiters on their shortest paths. A clear-cut distinction between small $\left(L_{\mathrm{c}} \approx 250 \mathrm{~m}\right)$ and 
large $\left(L_{\mathrm{c}} \geq 10 \mathrm{~km}\right)$ connection lengths is seen at the normalized radial positions $0.3,0.55$ and 0.7 , where the respective main resonances $q=12 / 2,13 / 2$ and 14/2 reside.

The effective radial plasma transport is governed by a complex 3D interaction between short and long flux tubes. Note that the vertical stripes with are about $3 \mathrm{~m}$ apart from each other, whereas their radial extension, the SOL depth, is about $10 \mathrm{~cm}$ in the average. The largest fraction of the power outflux from the core enters the SOL in the poloidal regions of large $L_{\mathrm{c}}$ between the vertical stripes of small $L_{\mathrm{c}}$. Its poloidal diffusion into the sink regions of the limiter shadow is quite ineffective due to the large poloidal distances between the stripes. This leads to a poloidal decoupling of all plasma parameters between the open and shadowed regions, which is reflected by the poloidal modulation of $T_{\mathrm{e}}$ in Fig. 3. However, the code predicts that more than $95 \%$ of the power entering the SOL is lost to the limiters, not to the wall, which is evidenced in Fig. 3 by the overall radial decay of the temperature. This means that there is a transport channel, different from poloidal diffusion, being responsible for the poloidal transfer of the power to the shadowed regions. It can be shown that this channel is parallel heat conduction, coupled with radial diffusion, in the radial layer between any two adjacent main resonances.

This power-loss channel to the short flux tubes limits the rise of the upstream plasma parameters on the long flux tubes and explains why the power-load distribution on the limiters is smooth (no hot spots!) and does not correlate with a strongly inhomogeneous distribution of connection lengths over the limiters [9]. Fine structures in the $L_{\mathrm{c}}$ distribution (Fig. 2) are also smoothed out by the cross-field heat transport across long flux tubes.

The main effect of the long flux tubes is simply to increase the radial decay lengths of the plasma parameters. This effect enlarges the power deposition area sufficiently to prevent the predicted peak power load from exceeding the range of the engineering limit of $5-8$ $\mathrm{MW} / \mathrm{m}^{2}$ even in the worst case of the assumed highest density and lowest heat conduction.

\section{TEXTOR-DED}

The Dynamic Ergodic Divertor (DED) has been installed in TEXTOR to improve the power exhaust and particle control by providing an active control of the plasma edge [4]. A complex 3D stochastic region is generated by resonant magnetic perturbations produced by helical conductors, installed on the inboard side of the vessel, having the same pitch angle as the field lines of the $q=3$ magnetic surface [14]. The DED coils are covered by a smooth surface of tiles acting as a target plate.

The computational domain for the plasma extends over the radial perturbation region of interest, the inner boundary being a closed magnetic surface, the outer boundary a magnetic surface of the non-perturbative case at the outermost position of the DED target [15]. The standard operational mode is 12/4. The corresponding DED current $\left(I_{\mathrm{DED}}\right)$ generates a sequence of resonances with poloidal numbers 9 to 14. Radially outwards the island chains break up and form an extended radial stochastic region of about $5 \mathrm{~cm}$ up to 
the target (Fig. 4). The distribution of connection lengths shows a typical "finger" pattern formed by the remnant legs of the broken islands (Fig. 5). In the present example, $I_{\mathrm{DED}}=$ $13.5 \mathrm{kA}$ has been chosen, which is close to the max. value of $15 \mathrm{kA}$. Generally higher $I_{\mathrm{DED}}$ generate larger islands, larger island remnants and larger stochastic regions.

In the non-perturbative case, the flux surfaces are intact and the plasma parameters are poloidally smooth up to the DED target, which acts as a limiter. With the DED currents switched on in the EMC3 simulations, the plasma parameters follow the poloidal modulation of the connection length distribution, reflecting the dominant role of the parallel transport $[15,16]$. At the very edge, the strong distortion of the field lines due to the near-coil field modulation generates significant radial field components leading to an enhancement of the effective radial heat transport near the target (Fig. 6). This in turn implies a drop of the local $T_{\mathrm{e}}$ gradients, with a consequent reduction of the temperature in the whole edge region. These code predictions have been confirmed qualitatively by the experiment [17]. The main energy outflow is channelled to the edge by long flux tubes, but near the target a certain fraction of the power is transferred by cross-field transport from the poloidally narrowing fingers of the long flux tube to the poloidally expanding regions of the short flux tubes. This fraction depends sensitively on the magnetic field topology and the chosen cross-field transport coefficients.

The predicted footprints of the particle deposition on the target is typically characterized by four stripes reflecting the poloidal number of the main resonance and the field modulation in the laminar zone. These code simulations have also been confirmed by the experiment, specifically by target-viewing $2 \mathrm{D} H_{\alpha}$ diagnostic data [17].

\section{W7-AS}

The third example is concerned with the island divertor of W7-AS. Here, an intrinsically 3D SOL is generated by $10 \mathrm{up} /$ down symmetric divertor targets and by a chain of large magnetic islands. The targets introduce periodic localized recycling zones and the toroidal variation of the island shape inhomogeneous radial transport fluxes. Both effects lead to a toroidal modulation of the plasma parameters along the islands, which cannot be smoothed out by parallel heat conduction [18].

Dedicated experimental and modelling investigations conducted in the past few years have clarified several essential features of the W7-AS island divertor physics $[19,20]$. Here, only the detachment issue is mentioned, which has been covered in the last phase of the W7-AS operation.

After the discovery of the HDH regime [21], partial stable detachment with very high separatrix densities and more than $90 \%$ of the SOL power being radiated at the edge has been achieved in the W7-AS island divertor. Energy detachment sets in when the impurity radiation capability at the target exceeds the SOL input power [18]. Then, the radiation layer lifts from the target and moves towards the $X$-point located in front of the target. The detachment becomes stable only if power transport and radiation are in balance at a position inside the island SOL. Both experiment and modeling have shown 
that this balance can only be established in a divertor geometry having large plasma-tocore distances and small connection lengths [22,23]. In these cases, the modeling predicts the formation, at detachment conditions, of a carbon radiation belt inside the islands at the HFS. On the other hand, the radiation-free power channel at the LFS maintains a warm divertor region, which sustains the recycling process and causes a local peak of the power load on the target (Fig. 7). This hot spot, which has been detected by target thermography, prevents the establishment of a complete stable detachment in W7-AS.

\section{Conclusion}

Three devices with strongly different non-axisymmetric SOL topologies and magnetic field structures have been selected to demonstrate the need and relevance of realistic 3D transport modeling for understanding the physics of 3D SOL plasma transport. In particular, the two presented tokamak examples show that a release of the axisymmery constraint by small material or magnetic perturbations may totally alter the transport characteristics of standard tokamaks. The EMC3-EIRENE code has been used to simulate self-consistently the plasma and neutral transport processes, with impurity transport being included whenever relevant (e.g. detachment in W7-AS). Major predictions have been confirmed by the experiments. For complex 3D topologies, physical averaging prescriptions capable of reducing the dimensionality of the transport processes in numerical models are presently not available. However, given the large computational effort required by full 3D simulations, simplified 1D radial models using free parameters fitted to the results of self-consistent $3 \mathrm{D}$ simulations may be attractive e.g. for highlighting global transport effects in the SOL or for improving the efficiency of largescale design-optimization studies. 


\section{References}

[1] F. Sardei, Y. Feng, P. Grigull et al., J. Nucl. Mater. 241-243 (1997) 135

[2] Technical Basis for the ITER Final Design 2001 ITER EDA, Documentation Series No. 24, IAEA, Vienna 2001

[3] K.H. Finken, G. Fuchs, B. Giesen et al., J. Nucl. Materials 220-222 (1995) 448

[4] K.H. Finken and G.H. Wolf, Fusion Eng. Design 37 (1997) 337

[5] A. Samain, A. Grosman, T. Blenski et al., J. Nucl. Mater. 128-129 (1984) 395

[6] Ph. Gendrih, H. Capes, F. Nguyen, A, Samain, Contrib. to Plasma Physics 32 (1992) 179

[7] T. Evans, R.A. Moyer, P.R.Thomas et al., Phys. Rev. Lett., 92 (2004) 235003

[8] R.A. Moyer, T. Evans, T.H. Osborne et al., Physics of Plasmas 12 (2005) 056119

[9] M. Kobayashi, Y. Feng, A. Loarte et al., Nucl. Fusion 47 (2007) 61

[10] Y. Feng, F. Sardei, J. Kisslinger, J. Nucl. Mat. 266-269 (1999) 812

[11] D. Reiter, Technical Report Jül-1947 (1984) KFA Jülich, Germany

[12] Y. Gribov, Control System Design and Assessment (CSD), Appendix B: Magnetic configuration and poloidal field scenarios, N 19 RI 14 R0.1, Aug. 2004

[13] G. Federici, O. Zolotukhin, A. Tanga et al., Simulations of ITER start-up for limiter power load assessment, ITER D 2229DE v1, Jan. 2006

[14] M.W. Jakubowski, S.S. Abdullaev, K.H. Finken et al., Nucl. Fusion 44 (2004) S1

[15] D. Harting, D. Reiter, Technical Report Jül-4173 (2005) KFA Jülich, Germany

[16] M. Kobayashi, Y. Feng, F. Sardei et al., Nucl. Fusion 44 (2004) S64

[17] O. Schmitz, D. Harting, S. Abdullaev et al., P3-30, this conference

[18] Y. Feng, F. Sardei, P. Grigull et al., Plasma Phys. Control. Fusion 44 (2002) 611

[19] P. Grigull, K. McCormick, J Baldzuhn et al., Plasma Phys. Control. Fusion 43 (2001) A175

[20] Y. Feng, F. Sardei, P. Grigull et al., Nucl. Fusion 46 (2006) 807

[21] K. McCormick, P. Grigull, R. Burhenn et al., Phys. Rev. Letters 89 (2002) 015001

[22] P. Grigull, K. McCormick, Y. Feng et al., J. Nucl. Mater. 313-316 (2003) 1287

[23] Y. Feng, F. Sardei, J. Kisslinger et al., Nucl. Fusion 45 (2005) 89 


\section{Figure captions}

Fig. 1: Computational domain (dashed lines) used in the EMC3-EIRENE code for the plasma-transport simulations in the 6.5 MA start-up limiter configuration of ITER.

Fig. 2: Distribution of connection lengths over the plasma computational domain.

Fig. 3: $T_{e}$ distribution over the computational domain for the 6.5 MA configuration. $P_{S O L}=6 \mathrm{MW}, n_{u p}=0.54 \times 10^{19} \mathrm{~m}^{-3}, D_{\perp}=0.4 \mathrm{~m}^{2} / \mathrm{s}, \chi_{\perp}=1.6 \mathrm{~m}^{2} / \mathrm{s}$. The fine structure of connection lengths shown in Fig. 2 is not reflected by $T_{e}$ except for the limiter shadow region induced by the main resonance.

Fig. 4: Poincaré plot of the TEXTOR-DED edge region, standard 12/4 mode, for a perturbation current of $13.5 \mathrm{kA}$. Plasma current $=400 \mathrm{kA}, P_{S O L}=600 \mathrm{~kW}, \beta_{p o l}=$ 0.23 . Obtained by field-line tracing (Gourdon code).

Fig. 5: Distribution of connection lengths over the plasma computational domain as obtained by field-line tracing (Gourdon code) . The red regions represent closed flux surfaces and islands.

Fig. 6: Radial profile of the effective radial heat transport coefficient, normalized to that of the unperturbed configuration.

Fig. 7: Typical distribution of carbon radiation and hydrogen ionization in a stable partial detachment for the W7-AS standard divertor configuration. $P_{S O L}=0.8 \mathrm{MW}, n_{u p}=$ $5.5 \times 10^{19} \mathrm{~m}^{-3}, D=0.5 \mathrm{~m}^{2} / \mathrm{s}, \chi=3 D$. A hot spot on the target is detected by thermography in agreement with code results. It originates from a radiation-free power channel on the LFS, as shown by the simulations. 


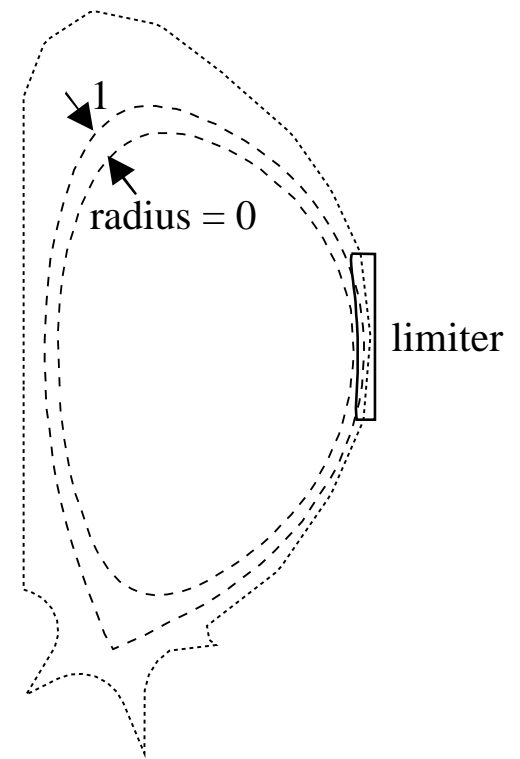

Fig. 1 


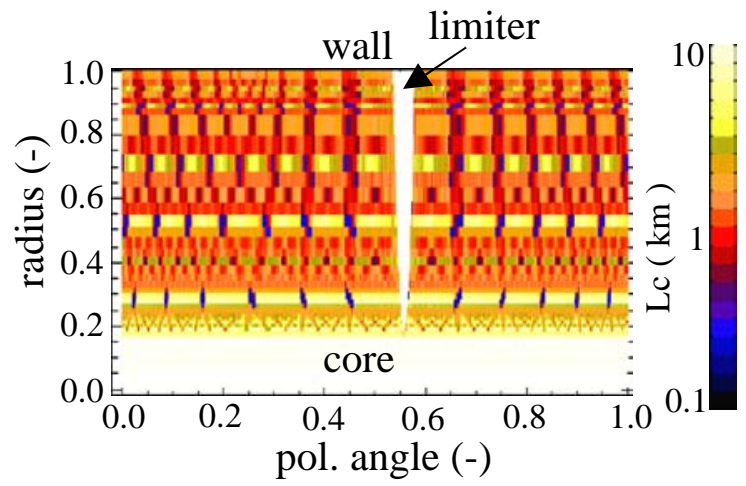

Fig. 2 


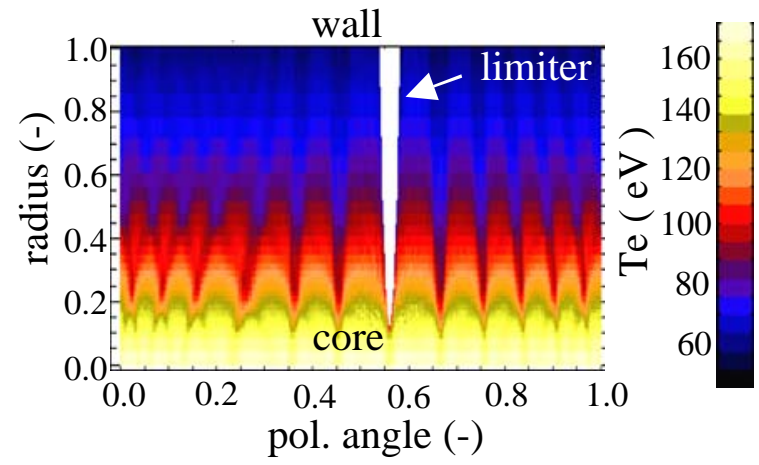

Fig. 3 


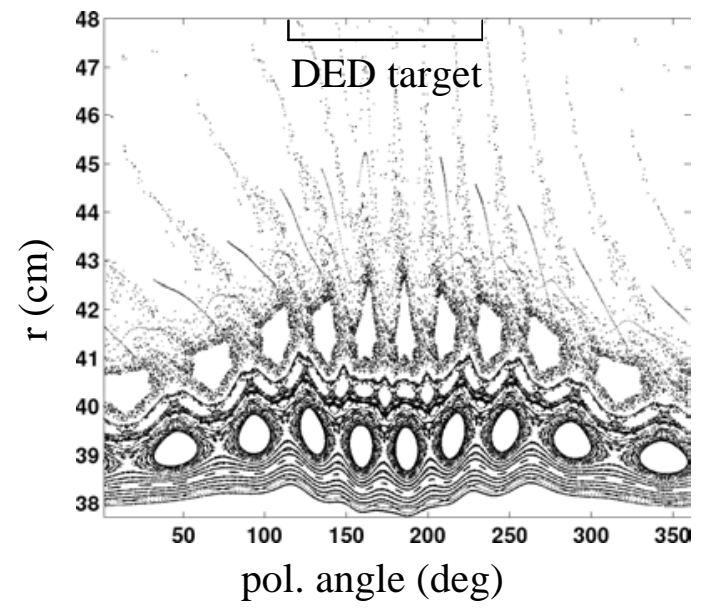

Fig. 4 


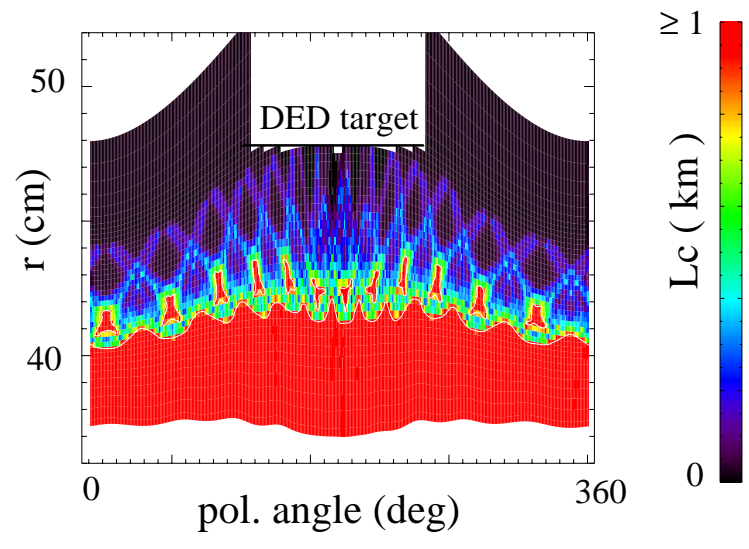

Fig. 5 


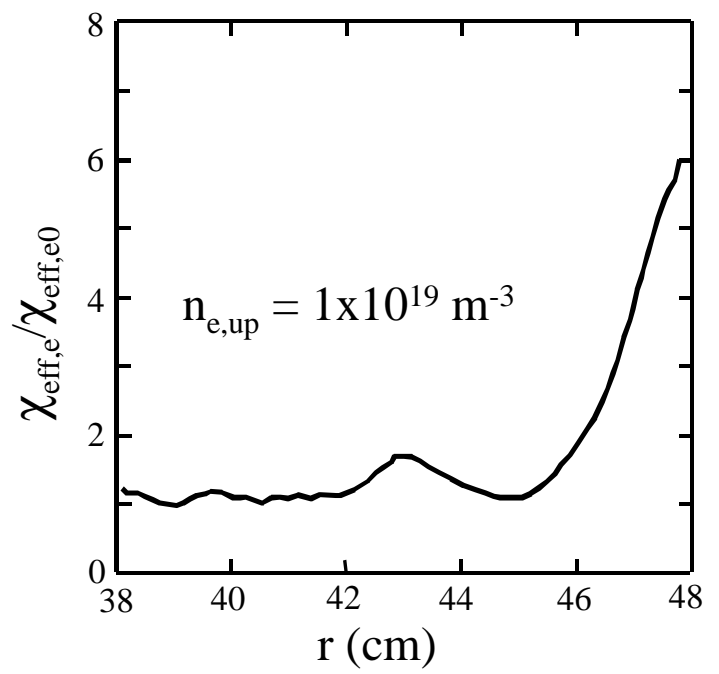

Fig. 6 


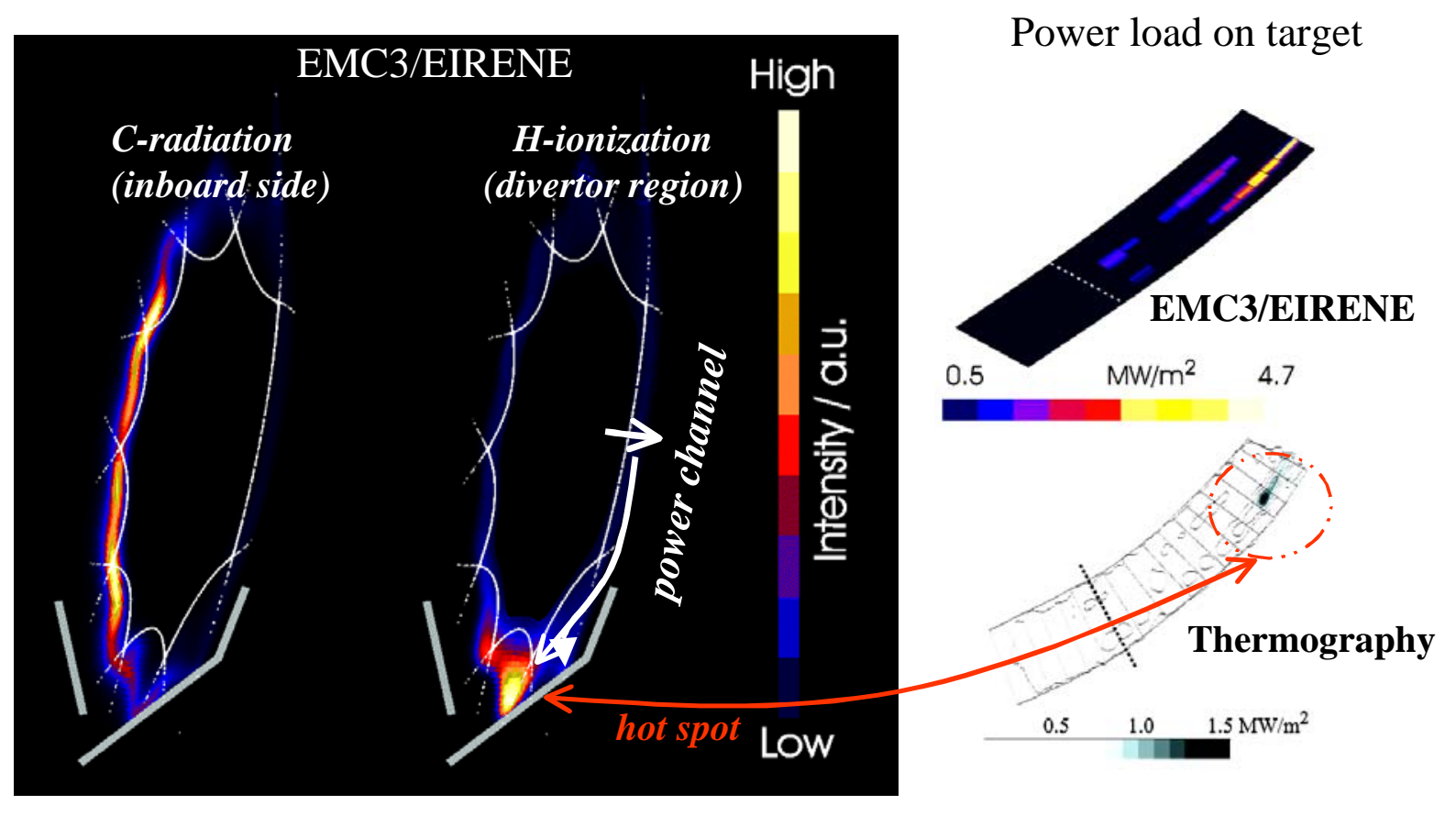

Fig. 7 